\title{
On Van, $r$ and $s$ topological properties of the Sierpinski triangle networks
}

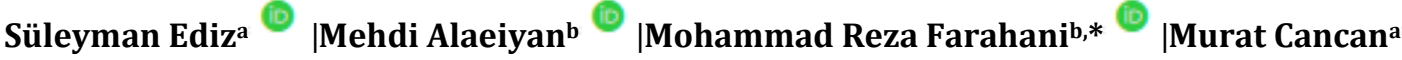

${ }^{a}$ Faculty of Education, Van Yuzuncu Yll A topological index-a numerical quantity derived from the graph University, Van, Turkey

${ }^{b}$ Department of Mathematics, Iran University of Science and Technology (IUST) Narmak, 16844, Tehran, Iran of a chemical network-is used for modelling the mathematical, chemical and physical properties of these networks and chemicals. The topological properties of the Sierpinski triangle has been newly studied in chemical graph theory. In this study we defined novel Van, $\mathrm{R}$ and $\mathrm{S}$ degree concepts as well as novel Van, $\mathrm{R}$ and $\mathrm{S}$ topological indices, and computed these topological indices for the Sierpinski triangle network. The closed formulas of these novel topological indices for the Sierpinski triangle network were presented.
\end{abstract}

\section{*Corresponding Author:}

Mohammad Reza Farahani

Email: Mr_farahani@mathdep.iust.ac.ir

Tel.: +98-919-2478265

\section{KEYWORDS}

$\mathrm{R}$ indices; $\mathrm{S}$ indices; Sierpinski triangle; Van indices.

\section{Introduction}

Mathematical chemistry is the area of research engaged in new application of mathematics in chemistry. Major areas of research in mathematical chemistry include chemical graph theory. Chemical graph theory applies graph theory to mathematical modeling of chemical phenomena. Chemical graph theory which is one of the most important branches of the applied mathematics has many applications related to the chemical and biological sciences. These chemical and biological applications of the graph theory contribute the birth of "chemo-informatics". Chemo-informatics is the intersection of the sciences of mathematics, chemistry and informatics. In chemistry, sometimes conducting the experimental studies of materials cannot be possible due to the expensiveness and complexity of large-scale materials such as chemical networks. In these situations theoretical studies are indispensable for modelling and predicting physicochemical and biological properties of such materials, molecules and networks. In chemo informatics literature these studies are named as "Quantitative structure-property and structure-activity relationships studies (QSPR and QSAR)". In QSAR $\backslash Q S P R$ studies, scientists need to understand the underlying topology of chemical networks via some numerical parameters derived from the molecular graphs of these networks. These numerical parameters which defined as topological descriptors are used for modelling and prediction of the physicochemical and biological features of chemical networks usually by means of correlation efficient. Among the all topological descriptors, the topological indices are most used parameters in QSAR \QSPR studies. Many molecular topological studies showed the importance of molecular topological descriptors in QSAR $\backslash Q S P R$ studies. Zagreb and Randić indices are the most used indices among the all topological indices in this regard Gutman and Trinajstić, (1972): Randić (1975) [1,2]. Fractals were introduced as the geometry of nature by Mandelbrot and studied in various fields including mathematics, social science, computer science, engineering, economics, 
physics, chemistry, and biology. Early in 1915, Sierpinski introduced a classic fractal which was called the Sierpinski triangle. The Sierpinski triangle is probably the most classical example of a self-similar fractal lattice: in contrast to the translational invariance of lattices such as the square lattice or the honeycomb, one of the main properties of self-similar lattices is scaling-invariance. Construction of the Sierpinski triangle can be defined in multiple ways. One of them is first consider the equilateral triangle denoted by $\mathrm{T}_{0}$. Subdivide $\mathrm{T}_{0}$ into four smaller congruent equilateral triangles and remove the central one, except for external points. We thus obtained three smaller equilateral subtriangles and denote this by $\mathrm{T}_{1}$ : After one more step, we get nine smaller equilateral subtriangles and denote this by $\mathrm{T}_{2}$. Continuing this process gives us the Sierpinski triangle (Figure 1).

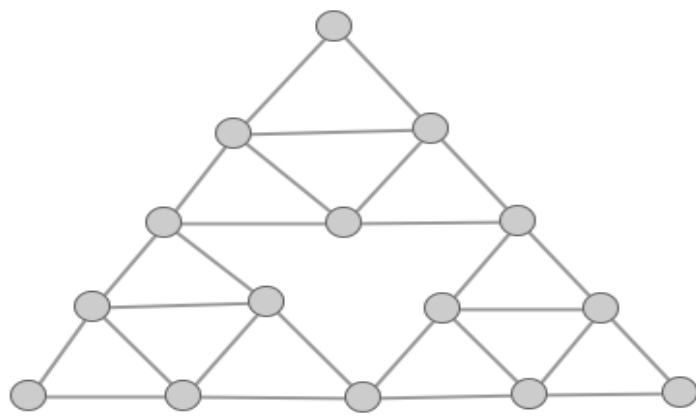

Figure 1 The fourth level of the Sierpinski triangle network: $\mathrm{S}_{4}$

For physical and electronic properties of the Sierpinski networks, we referred the interested reader to the references Ali, Rafique, Arshad et al. (2019): Jiang and Yang (2018) [3,4] and the references in these studies. For the mathematical properties of the Sierpinski networks we referred the interested reader to the references Saltan, Aslan and Demir (2019): Gu, Lau, Qiu (2019): Wang and Li (2019) [5-7].

Studies of the topological indices of the Sierpinski triangle have been made by Chen, He, Wang (2019): Liu, Zhao, He et al. (2019): Yamaç and Cancan (2019) and Siddiqui and
Baby (2016) [8-14]. The authors investigated the eccentric distance sum of Sierpinski triangle and Sierpinski networks, Zagreb type indices of Sierpinski triangle network and vedegree based topological indices of Sierpinski triangle network, respectively.

As a continuation of these studies, in this study we newly define and calculate Van, $\mathrm{R}$ and $\mathrm{S}$ indices of the Sierpinski triangle network.

\section{Preliminaries}

In this section we discuss some basic and preliminary definitions which we shall use later. A graph $G=(V, E)$ consists of two nonempty sets $V$ and 2-element subsets of $V$ namely $E$. The elements of $V$ are called vertices and the elements of $E$ are called edges. For a vertex $v, \operatorname{deg}(v)$ show the number of edges that incident to $v$. The set of all vertices which adjacent to $v$ is called the open neighborhood of $v$ and denoted by $N(v)$. For a vertex $v, S_{v}=$ $\sum_{u \in N(v)} \operatorname{deg}(u)$. For conveince, we name $S_{v}$ as "the sum degree of $v$ " or briefly "sum degree". For a vertex $v, \quad M_{v}=\prod_{u \in N(v)} \operatorname{deg}(u)$. For conveince, we name $M_{v}$ as "the multiplication degree of $v$ " or briefly "multiplication degree".

Definition 1 (Van degree) The Van degree of a vertex $v$ of a simple connected graph $G$ defined as:

$\operatorname{van}(v)=\frac{\sum_{u \in N(v)} \operatorname{deg}(u)}{\prod_{u \in N(v)} \operatorname{deg}(u)}=\frac{S_{v}}{M_{v}}$

Definition 2 (Reverse Van degree) The reverse Van degree of a vertex $v$ of a simple connected graph $G$ defined as:

$\operatorname{rvan}(v)=\frac{\prod_{u \in N(v)} \operatorname{deg}(u)}{\sum_{u \in N(v)} \operatorname{deg}(u)}=\frac{M_{v}}{S_{v}}$

Definition 3 (R degree) The $R$ degree of a vertex $v$ of a simple connected graph $G$ defined as:

$r(v)=M_{v}+S_{v}$

Definition 4 (reverse $\mathbf{R}$ degree) The $\mathrm{R}$ degree of a vertex $v$ of a simple connected graph $G$ defined as: 
$r r(v)=\frac{1}{M_{v}+S_{v}}$

Definition 5 (S degree) The $S$ degree of a vertex $v$ of a simple connected graph $G$ defined as:

$$
s(v)=\left|M_{v}-S_{v}\right|
$$

Definiton 6 (the first Van index) The first Van index of a simple connected graph $G$ defined as:

$$
\operatorname{Van}^{1}(G)=\sum_{v \in V(G)} \operatorname{van}(v)^{2}
$$

Definition 7 (the second Van index) The second Van index of a simple connected graph $G$ defined as:

$$
\operatorname{Van}^{2}(G)=\sum_{u v E(G)} \operatorname{van}(u) \operatorname{van}(v)
$$

Definition 8 (the third Van index) The third Van index of a simple connected graph $G$ defined as:

$$
\operatorname{Van}^{3}(G)=\sum_{u v \in E(G)}[\operatorname{van}(u)+\operatorname{van}(v)]
$$

Definition 9 (the first reverse Van index) The first reverse Van index of a simple connected graph $G$ defined as:

$$
\operatorname{Van}^{1 r}(G)=\sum_{v \in V(G)} r \operatorname{van}(v)^{2}
$$

Definition 10 (the second reverse Van index) The second reverse Van index of a simple connected graph $G$ defined as:

$$
\operatorname{Van}^{2 r}(G)=\sum_{u v E(G)} \operatorname{rvan}(u) \operatorname{rvan}(v)
$$

Definition 11 (the third reverse Van index)The third reverse Van index of a simple connected graph $G$ defined as:

$$
\operatorname{Van}^{3 r}(G)=\sum_{u v E(G)}[\operatorname{rvan}(u)+r v a n(v)]
$$

Definiton 12 (the first $\mathbf{R}$ index) The first $\mathrm{R}$ index of a simple connected graph $G$ defined as:

$$
R^{1}(G)=\sum_{v \in V(G)} r(v)^{2}
$$

Definition 13 (the second $\mathbf{R}$ index) The second R index of a simple connected graph $G$ defined as:

$$
R^{2}(G)=\sum_{u v \in E(G)} r(u) r(v)
$$

Definition 14 (the third R index) The third R index of a simple connected graph $G$ defined as:

$$
R^{3}(G)=\sum_{u v \in E(G)}[r(u)+r(v)]
$$

Definition 15 (the first reverse $\mathbf{R}$ index) The first reverse $\mathrm{R}$ index of a simple connected graph $G$ defined as:

$$
R^{1 r}(G)=\sum_{v \in V(G)} r r(v)^{2}
$$

Definition 16 (the second reverse $R$ index) The second reverse $R$ index of a simple connected graph $G$ defined as:

$$
R^{2 r}(G)=\sum_{u v \in E(G)} \operatorname{rr}(u) r r(v)
$$

Definition 17 (the third reverse $\mathbf{R}$ index): The third reverse $R$ index of a simple connected graph $G$ defined as:

$$
R^{3 r}(G)=\sum_{u v \in E(G)}[r r(u)+r r(v)]
$$

Definiton 18 (the first $\mathbf{S}$ index) The first $\mathrm{S}$ index of a simple connected graph $G$ defined as:

$$
S^{1}(G)=\sum_{v \in V(G)} s(v)^{2}
$$

Definition 19 (the second $\mathbf{S}$ index) The second $S$ index of a simple connected graph $G$ defined as:

$$
S^{2}(G)=\sum_{u v \in E(G)} s(u) s(v)
$$

Definition 20 (the third S index) The third S index of a simple connected graph $G$ defined as:

$$
S^{3}(G)=\sum_{u v \in E(G)}[s(u)+s(v)]
$$

After these novel indices definitions, we calculate the Van, $\mathrm{R}$ and $\mathrm{S}$ indices for the Sierpinski triangle network in the following section.

\section{Results and discussion}

We know that the Sierpinski triangle network has $1 / 2\left(3^{n}+3\right)$ vertices and $3^{n}$ edges. The edge partition of Sierpinski triangle network given in Table 1. 
TABLE 1 The degrees of the end vertices of edges for Sierpinski triangle $S_{n}$ network

\begin{tabular}{cc}
\hline $\left.\boldsymbol{( d e g}_{u}, \mathbf{d e g}_{\mathbf{v}}\right)$ & Number of Edges \\
\hline$(2,4)$ & 6 \\
$(4,4)$ & $3^{n-6}$ \\
\hline
\end{tabular}

From the Figure 1 and Table 1, the Van degree $\operatorname{van}(v)$ for the vertices of Sierpinski triangle $S_{n}$ network can be given in Table 2 .

TABLE 2 The Van degree van( $v$ ) partition for the vertices of Sierpinski triangle $S_{n}$ network

\begin{tabular}{cc}
\hline $\operatorname{van}(v)$ & Number of vertices \\
\hline $1 / 2$ & 3 \\
$\frac{7}{64}$ & 6 \\
$\frac{1}{16}$ & $1 / 2\left(3^{n}-15\right)$ \\
\hline
\end{tabular}

From the Figure 1 and Table 1, the reverse van degree $\operatorname{rvan}(v)$ for the vertices of Sierpinski triangle $S_{n}$ network can be given in Table 3 .

TABLE 3 The reverse Van degree rvan(v) partition for the vertices of Sierpinski triangle $S_{n}$ network

\begin{tabular}{cc}
\hline $\operatorname{rvan}(\boldsymbol{v})$ & Number of vertices \\
\hline 2 & 3 \\
$\frac{64}{7}$ & 6 \\
16 & $1 / 2\left(3^{n}-15\right)$ \\
\hline
\end{tabular}

The R degree $r(v)$ for the vertices of Sierpinski triangle $S_{n}$ networks presented in Table 4.

TABLE 4 The R degree $r(v)$ partition for the vertices of Sierpinski triangle $S_{n}$ network

\begin{tabular}{cc}
\hline $\boldsymbol{r}(\boldsymbol{v})$ & Number of vertices \\
\hline 24 & 3 \\
142 & 6 \\
272 & $1 / 2\left(3^{n}-15\right)$ \\
\hline
\end{tabular}

(From the Figure 1 and Table 1, the reverse $\mathrm{R}$ degree $\operatorname{rr}(v)$ for the vertices of Sierpinski triangle $S_{n}$ network can be given in Table 5.)
TABLE 5 The reverse R degree $\operatorname{rr}(v)$ partition for the vertices of Sierpinski triangle $S_{n}$ network.

\begin{tabular}{cc}
\hline $\boldsymbol{r} \boldsymbol{r}(\boldsymbol{v})$ & Number of vertices \\
\hline$\frac{1}{24}$ & 3 \\
$\frac{1}{142}$ & 6 \\
$\frac{1}{272}$ & $1 / 2\left(3^{n-15)}\right.$ \\
\hline
\end{tabular}

From the Figure 1 and Table 1 , the $\mathrm{S}$ degree $s(v)$ for the vertices of Sierpinski triangle $S_{n}$ network can be given in Table 6 .

TABLE 6 The $S$ degree $s(v)$ partition for the vertices of Sierpinski triangle $S_{n}$ network

\begin{tabular}{lc}
\hline \multicolumn{1}{c}{$\boldsymbol{s}(\boldsymbol{v})$} & Number of vertices \\
\hline 8 & 3 \\
114 & 6 \\
246 & $1 / 2\left(3^{n}-15\right)$ \\
\hline
\end{tabular}

From the Figure 1 and Table 1, the van degree $\operatorname{van}(v)$ for the end vertices of edges for Sierpinski triangle $S_{n}$ network can be given Table 7.

TABLE 7 The Van degrees of the end vertices of edges for Sierpinski triangle $S_{n}$ network

\begin{tabular}{cc}
\hline$(\operatorname{van}(u), \boldsymbol{v a n}(v))$ & Number of Edges \\
\hline$\left(\frac{1}{2}, \frac{7}{64}\right)$ & 3 \\
$\left(\frac{7}{64}, \frac{7}{64}\right)$ & 6 \\
$\left(\frac{7}{64}, \frac{1}{16}\right)$ & 12 \\
$\left(\frac{1}{16}, \frac{1}{16}\right)$ & $3^{n-21}$ \\
\hline
\end{tabular}

From the Figure 1 and Table 1, the reverse van degree $\operatorname{rvan}(v)$ for the end vertices of edges for Sierpinski triangle $S_{n}$ network can be given in Table 8. 
TABLE 8 The reverse van degrees of the end vertices of edges for Sierpinski triangle $S_{n}$ network

\begin{tabular}{cc}
\hline (rvan(u),rvan(v)) & Number of Edges \\
\hline$\left(2, \frac{64}{7}\right)$ & 6 \\
$\left(\frac{64}{7}, \frac{64}{7}\right)$ & 3 \\
$\left(\frac{64}{7}, 16\right)$ & 12 \\
$(16,16)$ & $3^{n}-21$ \\
\hline
\end{tabular}

From the Figure 1 and Table 1, the R degree $r(v)$ for the end vertices of edges for Sierpinski triangle $S_{n}$ network can be given in Table 9.

TABLE 9 The $\mathrm{R}$ degrees of the end vertices of edges for Sierpinski triangle $S_{n}$ network

\begin{tabular}{cc}
\hline $\boldsymbol{r}(\boldsymbol{u}), \boldsymbol{r}(\boldsymbol{v}))$ & Number of Edges \\
\hline$(24,142)$ & 6 \\
$(142,142)$ & 3 \\
$(272,142)$ & 12 \\
$(272,272)$ & $3^{n-21}$ \\
\hline
\end{tabular}

From the Figure 1 and Table 1 , the reverse $\mathrm{R}$ degree $\operatorname{rr}(v)$ for the end vertices of edges for Sierpinski triangle $S_{n}$ network can be given in Table 10.

TABLE 10 The reverse $\mathrm{R}$ degrees of the end vertices of edges for Sierpinski triangle $S_{n}$ network

\begin{tabular}{cc}
\hline$(\boldsymbol{r} \boldsymbol{r}(\boldsymbol{u}), \boldsymbol{r r}(\boldsymbol{v}))$ & Number of Edges \\
\hline$\left(\frac{1}{24}, \frac{1}{142}\right)$ & 6 \\
$\left(\frac{1}{142}, \frac{1}{142}\right)$ & 3 \\
$\left(\frac{1}{142}, \frac{1}{272}\right)$ & 12 \\
$\left(\frac{1}{272}, \frac{1}{272}\right)$ & $3^{n-21}$
\end{tabular}

The $S$ degree $s(v)$ for the end vertices of edges for Sierpinski triangle $S_{n}$ network is presented in Table 11.
TABLE 11 The $S$ degrees of the end vertices of edges for Sierpinski triangle $S_{n}$ network

\begin{tabular}{cc}
\hline $\boldsymbol{( s}(\boldsymbol{u}), \boldsymbol{s}(\boldsymbol{v}))$ & Number of Edges \\
\hline$(8,114)$ & 6 \\
$(114,114)$ & 3 \\
$(246,114)$ & 12 \\
$(246,246)$ & $3^{n}-21$ \\
\hline
\end{tabular}

Then, we begin to compute the Van, R and S topological indices for the Sierpinski triangle network.

Theorem 1 The first Van index of an arbitrary Sierpinski triangle network $S_{n}$ is equal to $3 x\left(\frac{1}{2}\right)^{2}+6 x\left(\frac{7}{64}\right)^{2}+\frac{1}{2}\left(3^{n}-15\right) x\left(\frac{7}{64}\right)^{2}$.

Proof From the definition of the first Van index and Tab. 2, we can directly write:

$\operatorname{Van}^{1}\left(S_{n}\right)=\sum_{v \in V\left(S_{n}\right)} \operatorname{van}(v)^{2}$

$=3 x\left(\frac{1}{2}\right)^{2}+6 x\left(\frac{7}{64}\right)^{2}+\frac{1}{2}\left(3^{n}-15\right) x\left(\frac{7}{64}\right)^{2}$

Theorem 2 The second Van index of an arbitrary Sierpinski triangle network $S_{n}$ is equal to $\frac{6}{2^{7}}+\frac{247}{2^{12}}+\frac{7}{2^{10}}+\frac{3^{n}-21}{2^{8}}$.

Proof From the definition of the second Van index and Tab. 7, we can directly write:

$\operatorname{Van}^{2}\left(S_{n}\right)=\sum_{u v \in E\left(S_{n}\right)} \operatorname{van}(u) \operatorname{van}(v)$

$=\frac{6}{2^{7}}+\frac{247}{2^{12}}+\frac{7}{2^{10}}+\frac{3^{n}-21}{2^{8}}$

Theorem 3 The third Van index of an arbitrary Sierpinski triangle network $S_{n}$ is equal to $\frac{234}{2^{6}}+\frac{21}{2^{5}}+\frac{132}{2^{6}}+\frac{3^{n}-21}{2^{3}}$.

Proof From the definition of the third Van index and Tab. 7, we can directly write:

$$
\begin{aligned}
& \operatorname{Van}^{3}\left(S_{n}\right)=\sum_{u v \in E\left(S_{n}\right)}(\operatorname{van}(u)+\operatorname{van}(v)) \\
& =\frac{234}{2^{6}}+\frac{21}{2^{5}}+\frac{132}{2^{6}}+\frac{3^{n}-21}{2^{3}}
\end{aligned}
$$

Theorem 4 The first reverse Van index of an arbitrary Sierpinski triangle network $S_{n}$ is equal to $3 x 2^{2}+6 x\left(\frac{64}{7}\right)^{2}+\frac{1}{2}\left(3^{n}-15\right) x 16^{2}$. 
Proof From the definition of the first reverse Van index and Tab. 3, we can directly write:

$$
\begin{aligned}
& \operatorname{Van}^{1 r}\left(S_{n}\right)=\sum_{v \in V\left(S_{n}\right)} r \operatorname{van}(v)^{2} \\
& =3 x 2^{2}+6 x\left(\frac{64}{7}\right)^{2}+\frac{1}{2}\left(3^{n}-15\right) \times 16^{2} .
\end{aligned}
$$

Theorem 5 The second reverse Van index of an arbitrary Sierpinski triangle network $S_{n}$ is equal to $6 x \frac{2^{7}}{7}+3 x \frac{2^{12}}{49}+12 x \frac{2^{10}}{7}+\left(3^{n}-21\right) x 2^{8}$.

Proof From the definition of the second reverse Van index and Tab. 8, we can directly write:

$$
\begin{aligned}
& \operatorname{Van}^{2 r}\left(S_{n}\right)=\sum_{u v \in E\left(S_{n}\right)} \operatorname{rvan}(u) r v a n(v) \\
& =6 x \frac{2^{7}}{7}+3 x \frac{2^{12}}{49}+12 x \frac{2^{10}}{7}+\left(3^{n}-21\right) \times 2^{8}
\end{aligned}
$$

Theorem 6 The third reverse Van index of an arbitrary Sierpinski triangle network $S_{n}$ is equal to $6 x \frac{78}{7}+3 x \frac{128}{7}+12 x \frac{176}{7}+\left(3^{n}-21\right) \times 32$.

Proof From the definition of the third reverse Van index and Tab. 8, we can directly write:

$$
\begin{aligned}
& \operatorname{Van}^{3 r}\left(S_{n}\right)=\sum_{u v \in E\left(S_{n}\right)}(r \operatorname{van}(u)+r \operatorname{van}(v)) \\
& =6 x \frac{78}{7}+3 x \frac{128}{7}+12 x \frac{176}{7}+\left(3^{n}-21\right) \times 32
\end{aligned}
$$

Theorem 7 The first R index of an arbitrary Sierpinski triangle network $S_{n}$ is equal to $3 \times 24^{2}+6 \times 142^{2}+\frac{1}{2}\left(3^{n}-15\right) \times 272^{2}$.

Proof From the definition of the first R index and Tab. 4, we can directly write:

$$
\begin{aligned}
& R^{1}\left(S_{n}\right)=\sum_{v \in V\left(S_{n}\right)} r(v)^{2}=3 \times 24^{2}+6 \times 142^{2} \\
& +\frac{1}{2}\left(3^{n}-15\right) \times 272^{2}
\end{aligned}
$$

Theorem 8 The second R index of an arbitrary Sierpinski triangle network $S_{n}$ is equal to $544428+\left(3^{n}-21\right) x 73984$.

Proof From the definition of the second $\mathrm{R}$ index and Tab. 9, we can directly write:

$$
\begin{aligned}
& R^{2}\left(S_{n}\right)=\sum_{u v \in E\left(S_{n}\right)} r(u) r(v) \\
& =6 \times 24 \times 142+3 \times 142 \times 142+12 \times 142 \times 272 \\
& +\left(3^{n}-21\right) \times 272 \times 272 \\
& =544428+\left(3^{n}-21\right) \times 73984 .
\end{aligned}
$$

Theorem 9 The third R index of an arbitrary Sierpinski triangle network $S_{n}$ is equal to $6816+\left(3^{n}-21\right) \times 544$.

Proof From the definition of the third R index and Tab. 9, we can directly write:

$$
\begin{aligned}
& R^{3}\left(S_{n}\right)=\sum_{u v \in E\left(S_{n}\right)}(r(u)+r(v)) \\
& =6 x 166+3 \times 284+12 \times 414+\left(3^{n}-21\right) \times 544 \\
& =6816+\left(3^{n}-21\right) \times 544
\end{aligned}
$$

Theorem 10 The first reverse $\mathrm{R}$ index of an arbitrary Sierpinski triangle network $S_{n}$ is equal to $3 x \frac{1}{24^{2}}+6 x \frac{1}{142^{2}}+\frac{1}{2}\left(3^{n}-15\right) \times \frac{1}{272^{2}}$. Proof From the definition of the first reverse $\mathrm{R}$ index and Tab. 5, we can directly write:

$$
R^{\operatorname{lr}}\left(S_{n}\right)=\sum_{v \in V\left(S_{n}\right)} r r(v)^{2}=3 x \frac{1}{24^{2}}+6 x \frac{1}{142^{2}}+\frac{1}{2}\left(3^{n}-15\right) x \frac{1}{272^{2}}
$$

Theorem 11 The second reverse R index of an arbitrary Sierpinski triangle network $S_{n}$ is equal to

$6 x \frac{1}{24} \times \frac{1}{142}+3 x \frac{1}{142} \times \frac{1}{142}+12 x \frac{1}{142} \times \frac{1}{272}+\left(3^{n}-21\right) x \frac{1}{272^{2}}$.

Proof From the definition of the second reverse $\mathrm{R}$ index and Tab. 10, we can directly write:

$$
\begin{aligned}
& R^{2 r}\left(S_{n}\right)=\sum_{u v \in E\left(S_{n}\right)} r r(u) r r(v) \\
& =6 \times \frac{1}{24} \times \frac{1}{142}+3 \times \frac{1}{142} \times \frac{1}{142}+12 \times \frac{1}{142} \times \frac{1}{272}+\left(3^{n}-21\right) \times \frac{1}{272} \times \frac{1}{272} .
\end{aligned}
$$

Theorem 12 The third reverse $\mathrm{R}$ index of an arbitrary Sierpinski triangle network $S_{n}$ is equal to $6816+\left(3^{n}-21\right) \times 544$.

Proof From the definition of the third reverse $\mathrm{R}$ index and Tab. 10, we can directly write:

$$
\begin{aligned}
& R^{3 r}\left(S_{n}\right)=\sum_{u v \in E\left(S_{n}\right)}(r r(u)+r r(v)) \\
& =6 x\left(\frac{1}{24}+\frac{1}{142}\right)+3 x\left(\frac{1}{142}+\frac{1}{142}\right)+12 x\left(\frac{1}{142}+\frac{1}{272}\right) \\
& +\left(3^{n}-21\right) x\left(\frac{1}{272}+\frac{1}{272}\right) \\
& =6 x\left(\frac{1}{24}+\frac{1}{142}\right)+3 x \frac{1}{71}+12 x\left(\frac{1}{142}+\frac{1}{272}\right)+\left(3^{n}-21\right) \times \frac{1}{136}
\end{aligned}
$$

Theorem 13 The first S index of an arbitrary Sierpinski triangle network $S_{n}$ is equal to $3 x 8^{2}+6 x 114^{2}+\frac{1}{2}\left(3^{n}-15\right) x 246^{2}$.

Proof From the definition of the first $\mathrm{S}$ index and Tab. 6, we can directly write: 
$S^{1}\left(S_{n}\right)=\sum_{v \in V\left(S_{n}\right)} s(v)^{2}=3 x 8^{2}+6 x 114^{2}+\frac{1}{2}\left(3^{n}-15\right) x 246^{2}$

Theorem 14 The second $S$ index of an arbitrary Sierpinski triangle network $S_{n}$ is equal to $380988+\left(3^{n}-21\right) \times 60516$.

Proof From the definition of the second S index and Table. 11, we can directly write:

$$
\begin{aligned}
& S^{2}\left(S_{n}\right)=\sum_{u v \in E\left(S_{n}\right)} s(u) s(v) \\
& =6 \times 8 \times 114+3 \times 114 \times 114+12 \times 114 \times 246 \\
& +\left(3^{n}-21\right) \times 246 \times 246 \\
& =380988+\left(3^{n}-21\right) x 60516
\end{aligned}
$$

Theorem 15 The third S index of an arbitrary Sierpinski triangle network $S_{n}$ is equal to $5736+\left(3^{n}-21\right) \times 592$.

Proof From the definition of the third S index and Tab. 11, we can directly write:

$$
\begin{aligned}
& S^{3}\left(S_{n}\right)=\sum_{u v \in E\left(S_{n}\right)}(s(u)+s(v)) \\
& =6 x(8+114)+3 x(114+114)+12 x(114+246) \\
& +\left(3^{n}-21\right) x(246+246) \\
& =6 x 122+3 x 228+12 \times 360+\left(3^{n}-21\right) x 592 \\
& =5736+\left(3^{n}-21\right) x 592 .+
\end{aligned}
$$

\section{Conclusion}

In this research study, the Van, $\mathrm{R}$ and $\mathrm{S}$ degree concepts and the Van, $\mathrm{R}$ and $\mathrm{S}$ topological indices were firstly defined using these novel degree notions. We assessed the Van, R and S topological indices of Sierpinski triangle network. The closed formulas of these novel indices for the Sierpinski triangle network were discussed. These closed formulas of the Sierpinski triangle network is crucial to understand the underlying toplogy of this network. The mathematical and chemical properties of Van, R and S topological indices of chemical structures are worth to study for future studies. In addition, evaluating the relationship of Van, R and S degree concepts with the other graph invariants will be an interesting research study.

\section{Orcid:}

Süleyman Ediz: https://orcid.org/0000-00030625-3634

Mehdi Alaeiyan: https://orcid.org/0000-00032185-5967

Mohammad Reza Farahani:

https://orcid.org/0000-0003-2969-4280

Murat Cancan: https://orcid.org/0000-00028606-2274

\section{Acknowledgments}

The authors would like to thank the referees and editors for their valuable comments and suggestions which helped to improve the manuscript.

\section{References}

[1] I. Gutman, N. Trinajstić, Chem. Phys. Let., 1972, 17, 535-538.

[2] M. Randić, J. Am. Chem. Soc. 1975, 97, 66096615.

[3] A.A. Ali, H. Rafique, T. Arshad, M.A. Alqarni, S. H. Chauhdary, A.K. Bashir, Sensors, 2019, 19, 678-695.

[4] Z. Jiang, W. Yan, J. Stat. Phys., 2018, 172, 19.

[5] M. Saltan, N. Aslan, B. Demir, Turk. J. Math., 2019, 43, 361-372.

[6] Q. Gu, K.S. Lau, H. Qiu, H. J. Math. Anal. App., 2019, 474, 674-692.

[7] Q. Wang, J.L Li, Math. Nachrichten, 2019, 292, 211-226.

[8] J. Chen, L. He, Q. Wang, Fractals, 2019, 27, 1950016 (8 pages).

[9] J.B. Liu, J. Zhao, H. He, Z. Shao, J. Stat. Phys., 2019, 177, 1131-1147.

[10] K. Yamaç, M. Cancan, Sigma J Eng \& Nat Sci., 2019, 37, 1271-1276.

[11] H.M.A Siddiqui, S. Baby, Global J. Math., 2016, 8, 793-801.

[12] M. Cancan, S. Ediz: M.R. Farahani, Eurasian Chem. Commun, 2020, 2, 641-645. 
[13] M. Alaeiyan, C. Natarajan, G. Sathiamoorthy, M.R. Farahani, Eurasian Chem. Commun, 2020, 2, 646-651.

[14] J. Liu, A. Baig, M. Imran, W. Khalid, M. Saeed, M.R. Farahani, Eurasian Chem. Commun, 2020, 2, 672-679.
How to cite this article: Süleyman Ediz, Mehdi Alaeiyan, Mohammad Reza Farahani*, Murat Cancan. On van, R and S topological properties of the Sierpinski triangle networks. Eurasian Chemical Communications, 2020, 2(7), 819-826. Link:

http://www.echemcom.com/article_1075 26.html

Copyright (C) 2020 by SPC (Sami Publishing Company) + is an open access article distributed under the Creative Commons Attribution License, which permits unrestricted use, distribution, and reproduction in any medium, provided the original work is properly cited. 